\title{
Lenguas de signos en el estado español: estatus y territorialidad
}

\author{
Carmen Cabeza Pereiro \\ Universidade de Vigo \\ cabeza@uvigo.es
}

Francisco Eijo Santos

Universidade de Vigo / Asociación de Sordos de Vigo

manos2_2ojos@hotmail.com

Recibido o 05/10/2017

Aceptado o 14/04/2018

\section{Sign languages in Spain: status and territoriality}

\section{Resumo}

El propósito de este artículo es presentar la situación de las lenguas de signos en el contexto de la política lingüística española, tanto en lo que se refiere al ámbito estatal como en el de las comunidades autónomas. Para ello se hace una revisión de las leyes que, en el ámbito estatal y autonómico, regulan en alguna medida el uso de estas lenguas. Trataremos de mostrar cómo el desarrollo normativo de las lenguas de signos (LS) se enfrenta en España a dos retos: el primero tiene que ver con el prejuicio de que las LS son adaptaciones para facilitar la accesibilidad a las personas sordas, y no lenguas naturales que se utilizan en un contexto social y cultural específico; el segundo proviene de la concepción territorial de los derechos lingüísticos. Solo la lengua de signos catalana y la lengua de signos española gozan de reconocimiento en el texto normativo de mayor rango, la primera en Cataluña y la segunda en el resto del estado, en tanto que las comunidades autónomas tienen la potestad de reconocer lenguas de signos propias de los respectivos territorios. En el caso gallego, una entrevista realizada al presidente de la Federación de Persoas Xordas de Galicia (FAXPG) nos ha permitido concluir que no está entre las prioridades de este órgano de representación la reivindicación de una lengua de signos gallega.

\section{Palabras chave}

Política lingüística; lengua de signos española; lengua de signos catalana; lengua de signos gallega

\section{Sumario}

1. Introducción. 2. Contexto de partida: la comunidad sorda y la Ley $27 / 2007$. 3. El modelo de la discapacidad y el de la reivindicación identitaria. 4. Política y planificación lingüística de las lenguas de signos. 4.1. Política lingüística y comunidades sordas. 4.2. La comunidad sorda española como agente de planificación. 5. La planificación de las lenguas de signos en España: consecuencias de la territorialidad. 6. El caso de Galicia: ¿lengua de signos española o lengua de signos gallega? 6.1. Sobre la oportunidad de reconocer una lengua de signos gallega. 6.2. Sobre las lenguas de signos en el sistema educativo. 6.3. Sobre las necesidades de la comunidad sorda gallega en términos de política lingüística 7. Recapitulación y reflexión final.
The purpose of this article is to present the situation of sign languages in the context of Spanish language policy, both at the state level and at the autonomous comunities level. The state and regional legislation that regulates to some extent the use of these languages is reviewed. The normative development of sign languages (SL) faces two challenges in Spain: one has to do with the incorrect belief that SLs are adaptations to facilitate accessibility for the Deaf, rather than natural languages used in a specific social and cultural context. The second challenge arises from the territorial conception of linguistic rights. Only Catalan Sign Language and Spanish Sign Language are recognized in the higher-level normative text, the first in Catalonia and the second in the rest of the state, while the Autonomous Communities have authority to recognize sign languages of their own territories. In the Galician case, an interview with the President of the Federation of Galician Deaf People has led us to conclude that recognition of a Galician Sign Language is not a priority for this representative body.

Keywords Language policy; Spanish Sign Language; Catalan Sign Language; Galician Sign Language

\section{Contents}

1. Introduction. 2. Background: the Deaf community and the Law 27/2007. 3. The impairment model and the identity recognition model. 4 . Sign languages policy and sign language planning. 4.1. Language policy and Deaf communities. 4.2. Spanish Deaf community as a planning agent. 5 Sign language planning in Spain: the consequences of territoriality. 6 . The case of Galicia: Spanish Sign Language or Galician Sign Language? 6.1. On the opportunity of recognizing a Galician Sign Language. 6.2. On sign languages in the education system. 6.3. On the linguistic needs of the Galician Deaf community. 7. Summary and final thoughts. 


\section{INTRODUCCIÓN}

El propósito de este artículo es presentar la situación de las lenguas de signos en el contexto de la política lingüística española, tanto en lo que se refiere al ámbito estatal como en el de las comunidades autónomas. Intentaremos explicar cuáles son los obstáculos que dificultan el desarrollo de los derechos lingüísticos de las personas usuarias de estas lenguas teniendo en cuenta el principio de territorialidad que rige en el estado. Tal principio, establecido para las lenguas orales cooficiales en las comunidades autónomas que así lo han establecido en sus estatutos, plantea para las formas de transmisión signada que usan las personas sordas una situación peculiar. Ha de tenerse en cuenta que las lenguas de signos o de señas son lenguas articuladas en una modalidad visogestual, pero estructuralmente independientes de las lenguas vocales con las que conviven, con las que tampoco tienen una relación genealógica (para entendernos: pertenecen a familias lingüísticas diferentes). El hecho de que se reconozca una lengua de signos que tiene como ámbito territorial el conjunto del estado salvo Cataluña (con una lengua de signos propia reconocida, como se verá), deja al arbitrio de cada una de las comunidades autónomas que se regulen o no los derechos de las personas sordas signantes en cada territorio. Esto conduce, como se tratará de explicar, a un tratamiento desigual en la práctica, ya que únicamente Cataluña ha desarrollado una política lingüística a favor de su lengua de signos propia. Las restantes comunidades autónomas que han incluido alguna mención a la(s) lengua(s) de signos en sus normativas consideran los derechos lingüísticos de las personas signantes como un aspecto de la discapacidad, y no como un asunto de política lingüística, lo cual explica en buena parte ese tratamiento inicuo. Ante esta situación, el dilema de la Federación de Persoas Xordas de Galicia, cuya postura analizamos en este trabajo, se resuelve a favor de una política lingüística estatal y con la renuncia a reivindicar una lengua de signos gallega.

El apartado 2 enmarca brevemente el problema de la comunicación signada en el contexto de la transmisión intergeneracional y presenta sumariamente la situación sociolingüística actual de la comunidad sorda en España. La sección 3 formula una dicotomía sobre la sordera, que va más allá de una cuestión conceptual, pues determina la forma de vivir de las personas sordas y la consideración de las lenguas de signos como verdaderas lenguas naturales. El apartado 4 introduce las singularidades de la política lingüística en las comunidades sordas, y más particularmente el caso de la comunidad sorda española. Es en la sección 5 donde abordamos la cuestión del modelo territorial español en relación con las lenguas de signos, fijándonos sobre todo en cuál es el tratamiento que se da a la(s) lengua(s) de signos en las diferentes normativas, con el objeto de valorar en qué medida la cuestión de las lenguas de signos corre pareja a la de las lenguas orales, es decir, si se plantea o no en el ámbito de los derechos lingüísticos de la ciudadanía. El apartado 6 se centra en el caso de Galicia, y en él se presentan las respuestas a una entrevista con el presidente de la Federación de Asociacións de Persoas Xordas de Galicia, en relación con el posible reconocimiento de una lengua de signos gallega. Por último, el apartado 7 ofrece una recapitulación y una serie de reflexiones finales.

\section{Contexto de partida: LA COMUnidAD SORDA y LA Ley 27/2007}

Aunque una parte relevante de los casos de sordera tiene una causa genética (alrededor de un $60 \%$ de las sorderas infantiles tienen un origen genético, según Marco / Matéu 2003), el porcentaje de personas sordas que nacen y crecen en una familia en la que alguno de los progenitores es sordo ronda el 5\% (Mitchell / Karchmer 2004). Estos progenitores sordos pueden ofrecer un input lingüístico en una lengua de modalidad visogestual, más concretamente, una de las llamadas lenguas de signos que suelen utilizar las personas sordas. Esto supone que para el $95 \%$ restante el acceso a una lengua nativa va a estar fuertemente condicionado por las condiciones personales y sociales para el aprendizaje, dándose el caso de que la falta de audición supone una barrera para la modalidad lingüística auditiva, aunque no para la modalidad visual, como se acaba de sugerir.

Esta circunstancia crea una singularidad en la forma de transmisión intergeneracional de las lenguas de signos, que se encuentra limitada, en su forma natural, al 5\% mencionado. El resto 
de las personas sordas signantes aprenden la lengua de signos en el colegio, cuando entran en contacto con otros individuos sordos, o incluso en etapas más tardías, ya en la adolescencia. El colegio tiene una función importantísima en esa transmisión?.

En el caso de los adultos sordos, tradicionalmente era la asociación de sordos el contexto en que se desarrollaban buena parte de sus prácticas comunicativas. Hoy en día, a esos espacios de encuentro tradicionales les están ganando terreno las redes sociales y otras formas de comunicación digital (videoconferencia o blog, por ejemplo) que permiten el uso de vídeo². En países como España, la comunidad sorda constituye una minoría lingüística fuertemente cohesionada por las lenguas de signos, que reconocen como símbolos de identidad grupal (Morales 2008; Jarque 2012), además de ser instrumentos de comunicación de uso cotidiano.

La ley $27 / 2007^{3}$ confirió una serie de derechos lingüísticos a las personas sordas, en particular en el ámbito educativo, en el acceso a servicios públicos tales como la salud, los transportes o las actividades culturales, deportivas y el ocio, así como también en las relaciones con la administración pública, la participación política y el acceso a los medios de comunicación. Reconoce dos lenguas de signos españolas, llamadas lengua de signos española (LSE) y lengua de signos catalana (LSC), y abre la posibilidad de que las comunidades autónomas (en adelante, CCAA), en el ámbito de su competencia, reconozcan otras lenguas de signos propias. La ley trata de forma paritaria a las personas sordas usuarias de una lengua de signos y a aquellas que optan por ayudas técnicas a la comunicación oral. A este segundo grupo les garantiza también el acceso a los recursos que requieren para la modalidad lingüística vocal que eligen. La misma ley dispone la creación del Centro de Normalización Lingüística de la Lengua de Signos Española, así como también del Centro Español del Subtitulado y Audiodescripción. El primero viene a satisfacer la necesidad de proteger y difundir la LSE, el segundo ha de contribuir a que se garantice el acceso a la información a las personas sordas que requieran ayudas técnicas en el contexto de los medios de apoyo a la comunicación oral.

En el seno de la comunidad signante, se han detectado opiniones negativas (Cabeza-Pereiro / Ramallo 2016) que pueden ser indicio de que no se han cumplido las expectativas que suscitó la aprobación de la ley. A principios del año 2017, el gobierno de España, a través del Ministerio de Sanidad, Asuntos Sociales e Igualdad, iniciaba un proceso de conversaciones para desarrollar normativamente la Ley $27 / 2007$. En concreto, convocaba a una serie de asociaciones de sordos, discapacitados auditivos, sordociegos, así como a otros grupos interesados en tal desarrollo normativo, tales como asociaciones que agrupan a padres y tutores de personas sordas o sordociegas, a personas con implante coclear o a profesionales de la interpretación de las lenguas de signos ${ }^{4}$. En marzo de 2018, una vez que el proyecto de Real Decreto está en periodo de alegaciones, la Confederación Estatal de Personas Sordas (CNSE) considera el texto insuficiente y solicita cambios que desarrollen adecuadamente la Ley 27/2007 y recojan las aspiraciones de la comunidad sorda en aspectos como el acceso a la información o la introducción de las lenguas de signos españolas como lenguas vehiculares en la educación ${ }^{5}$.

\footnotetext{
'La progresiva incorporación de intérpretes de lengua de signos en los colegios está contribuyendo a este aprendizaje de la lengua de signos por una parte de la población sorda en una forma y dimensión que requiere ser estudiada.

${ }^{2}$ También se echa de menos una investigación sobre estos cambios en las formas de comunicación y sobre el poder transformador que tienen en lo que se refiere al uso de variantes y repertorios lingüísticos.

${ }^{3}$ Ley 27/2007, de 23 de octubre, por la que se reconocen las lenguas de signos españolas y se regulan los medios de apoyo a la comunicación oral de las personas sordas, con discapacidad auditiva y sordociegas. Boletín Oficial del Estado, número 255, Madrid, 24 de octubre de 2007.

${ }^{4}$ Según una nota de prensa facilitada por el Ministerio de Sanidad, Asuntos Sociales e Igualdad, con el título "Primera reunión del grupo de trabajo para desarrollar la ley de lengua de signos", disponible en https://www.msssi.gob.es/gabinete/ notasPrensa.do?id=4075 (con acceso 6/7/2017), las entidades convocadas fueron: Confederación Española de Familias de Personas Sordas (FIAPAS), Asociación Española de Familias con Personas con Sordoceguera (APASCIDE), Federación Española de Sordoceguera (FESOCE), Fundación ONCE para la Atención de Personas con Sordoceguera (FOAPS), Confederación Estatal de Personas Sordas (CNSE), Centro de Normalización Lingüística de la Lengua de Signos Española (CNLSE), Federación de Asociaciones de Implantados Cocleares de España (AICE), Federación de Asociaciones de Personas Sordociegas de España (FASOCIDE) y Federación Española de Intérpretes de Lengua de Signos y Guías Intérpretes (FILSE).

${ }^{5}$ Ver "Comunicado de la presidenta de CNSE sobre el Reglamento de la Ley 27/2007", en http://www.cnse.es/home_detalle. php?id_inicio=164 (con acceso 16/3/2018).
} 
En este contexto se desarrolla el presente artículo, con el objetivo de identificar las causas que limitan el desarrollo de políticas lingüísticas que velen por un auténtico reconocimiento de los derechos lingüísticos de las personas sordas signantes que viven en España. La comunidad sorda española ha sido muy activa y reivindicativa en lo que se refiere al reconocimiento normativo de las lenguas de signos españolas, a su difusión y a la creación de materiales para su enseñanza. Es preciso tener en cuenta que estas acciones se han desarrollado en un contexto previamente definido para las lenguas orales cooficiales, en el cual los gobiernos autonómicos tienen capacidad de decidir sobre las lenguas y de implementar políticas lingüísticas. En tal contexto, la cuestión de la identidad sorda y su posible vinculación con una identidad nacional no es baladí, como no lo es tampoco el nombre que designa a las variedades signadas que se utilizan en cada territorio.

\section{El MODELO DE LA DISCAPACIDAD Y EL DE LA REIVINDICACIÓN IDENTITARIA}

Es habitual en los estudios sobre lenguas de signos y comunidades lingüísticas de sordos el confrontar dos modelos de representar conceptualmente la sordera y, por ende, las personas sordas (Baker-Shenk / Cokely 1980; Liñares Barbeito 2003): el modelo de la discapacidad o modelo clínicopatológico y el lingüístico o socioantropológico. El primero toma como punto de referencia a los individuos no afectados ("normales") para tratar de reparar o rehabilitar aquello que falta en los que tienen sordera. El segundo destaca la singularidad de las comunidades de sordos como grupos humanos que han desarrollado una forma de comunicación propia y, en torno a ella, todo un universo cultural y simbólico. La vida diaria de las personas sordas se ve afectada por este conflicto entre formas de vivir una identidad sorda, pero las soluciones particulares son diferentes entre sí, de tal manera que algunas de estas personas sienten un compromiso fuerte con la lengua de signos y la cultura sorda, y son altamente reivindicativas, en tanto que otras adoptan prácticas comunicativas mixtas o de compromiso.

Massone y Fojo dicen de las personas sordas en Argentina que

[C]onforman una comunidad lingüística que ha creado históricamente sus propias lenguas transmitidas en una modalidad visoespacial, que mantiene sus propios patrones de interacción social y cultural, y que está integrada económicamente pero separada de la sociedad de oyentes hablantes de español [...], hechos que han generado durante estos últimos 130 años prácticas sociales propias de la discriminación (Massone / Fojo 2011: 83).

Estos autores definen la lengua de signos argentina (LSA) como la lengua del endogrupo, en tanto que el español funcionaría, siempre según ellos, como una especie de lingua franca, necesaria e imperativa, puesto que es la lengua oficial de su país (Massone / Fojo 2011: 83).

La comunidad sorda española ha manejado el concepto de bilingüismo a lo largo de los últimos años, ya con anterioridad a la aprobación de la Ley 27/2007, como parte de un programa que habría de conducir a una adquisición de derechos lingüísticos en alguna de las lenguas de signos españolas. Un ejemplo es cómo se presentan los modelos educativos bilingües en el Libro Blanco de la Lengua de Signos Española en el Sistema Educativo:

Independientemente de la modalidad educativa, en algunos centros se ha adoptado el modelo bilingüe como seña de identidad de su Proyecto Educativo y Lingüístico, con principios básicos como el mismo estatus para las dos lenguas, la importancia de la coexistencia en el aula y en el centro de las dos lenguas y profesionales Sordos/as y oyentes como referentes lingüísticos y culturales de cada una de las lenguas (Alonso Baixeras et al. 2003: 29$)^{6}$.

Las prácticas de discriminación de las que hablan Massone y Fojo son resultado de la visión de la sordera como discapacidad y, como consecuencia de ello, de las personas sordas como individuos incompletos, sin tener en cuenta su especificidad lingüística y cultural. Esta visión implica, en la práctica, minorizar el valor instrumental de la lengua de signos y la cultura sorda, y por lo tanto trata de anular ambas (lengua y cultura), en lugar de tolerarlas.

\footnotetext{
${ }^{6}$ La práctica de escribir Sordo (con mayúscula) ha penetrado con fuerza en la comunidad sorda española. Las personas sordas de países anglófonos reivindican con la mayúscula Deaf la pertenencia a un grupo humano con una lengua propia. La ortografía del español no utiliza la mayúscula para designar una lengua o un pueblo, de ahí que se pierda esa diferencia entre tener la sordera como un atributo (sordo) frente a vivir la sordera como una forma de identidad (Sordo).
} 
Por el contrario, la perspectiva lingüística prioriza el papel de las lenguas de signos como instrumentos de comunicación, pues como tales funcionan en las prácticas comunicativas diarias de las personas sordas. Estas, además, constituyen auténticas comunidades lingüísticas en la medida en que son conscientes del valor de cohesión que, tanto a nivel funcional como en el plano simbólico, tiene la lengua de signos (Morales 2008).

\section{Política y planificación lingüística de las lenguas de signos}

\subsection{Política lingüística y comunidades sordas}

Hacer política lingüística equivale, entre otras cosas, a tomar decisiones sobre qué lenguas o qué variedades deben tenerse en cuenta para ser usadas en ámbitos de la vida pública. Este tipo de decisiones tiene consecuencias innegables para los usuarios y usuarias de esas lenguas o variedades, ya que o bien habilita la que usan de forma más natural como válida en diferentes esferas de la vida pública, o bien les exige acceder a otra para ejercer todos los derechos para los cuales se presupone el dominio de tal lengua o variedad oficial. Como casi todas las comunidades humanas son bilingües o multilingües (Romaine 2008), el hecho de tener que aprender una lengua mayoritaria para acceder al conocimiento y a la información es una situación muy común.

En el caso de las personas sordas esta cuestión tiene una dimensión especial, ya que su condición física les impide el acceso directo a la información proporcionada en lenguas orales, de tal manera que las hace dependientes de los intérpretes o de otros recursos lingüísticos de apoyo.

Reconocer esta situación es crucial, y también es crucial identificar la naturaleza lingüística del problema. Las personas sordas están plenamente capacitadas para hablar otra(s) lengua(s), las lenguas de signos, y en tanto que hablantes/signantes no tienen dificultades para disfrutar de experiencias comunicativas plenas. Aspiran al bilingüismo de forma reivindicativa porque no son efectivamente libres de elegir en qué código comunicarse. Solo desde el discurso del bilingüismo pueden ver reconocida su lengua.

De ahí que las reivindicaciones de las comunidades sordas se hayan dirigido de manera preferente, en diferentes entornos geográficos, al reconocimiento del estatus de la lengua, normalmente entendido como un reconocimiento legal, siempre al más alto nivel que se pueda alcanzar y, en segunda instancia, a su estandarización, para unificarla, difundirla y adaptarla a las necesidades del mundo moderno. Es el caso de la comunidad sorda española, como veremos.

Entre los países que tienen reconocida su lengua de signos nacional en la constitución están (De Meulder, 2015): Austria (2005), Finlandia (1995), Hungría (2011), Kenia (2010), Nueva Zelanda (2006), Sudáfrica (1996) o Zimbaue (2010). Portugal (1997) también tiene una mención a la lengua de signos portuguesa a propósito de cuestiones educativas. Además, Venezuela (1999) y Ecuador (2008) reconocen sus lenguas de signos como derechos derivados de la discapacidad. La autora añade a la lista otros países con algún tipo de reconocimiento legal para sus lenguas de signos. Los agrupa de la siguiente manera:

- Reconocen su lengua de signos nacional en el texto constitucional

- La reconocen en legislación general sobre lenguas

- La reconocen por una ley de lengua de signos

- La reconocen por una ley de lengua de signos que incluye también otros medios de comunicación (es en este apartado donde sitúa el caso de España)

- La reconocen en los estatutos de funcionamiento de un órgano de asesoramiento lingüístico

Los procesos de planificación de las lenguas de signos suelen tender hacia la conformación de lenguas de signos nacionales, no obstante, es habitual que algunas particularidades de convivencia imperantes en el seno de la sociedad general se reproduzcan también en el contexto de la comunidad sorda. Es el caso, por ejemplo, de la existencia de una lengua de signos de la provincia de Quebec (distinta de la utilizada por parte de las personas sordas de la parte an- 
glófona de Canadá (Parisot / Rinfret 2012), o de dos lenguas de signos en Finlandia (una de las cuales asociada a la cultura sueco-finesa, vid. De Meulder 2017). Reconocer la similitud entre los procesos de planificación de las LS y los desarrollados para lenguas orales minoritarias no es nuevo (De Meulder 2017).

\subsection{La comunidad sorda española como agente de planificación}

Como ya se ha adelantado, la comunidad sorda española ha impulsado desde hace décadas la reivindicación de los derechos lingüísticos, haciendo política lingüística según el modelo llamado «bottom-up» (de abajo-arriba, Gras 2004; Ferreiro / Aroca 2008; De Meulder 2017). Instituciones como la CNSE, la Fundación CNSE y, más recientemente, el Centro de Normalización Lingüística de la LSE (CNLSE), han desarrollado una labor de interlocución con el poder legislativo y con las administraciones centrales. En el caso de las CCAA, son las federaciones de personas sordas de cada territorio las que trabajan para mejorar las condiciones de vida de las personas signantes. Estos esfuerzos desembocaron en la promulgación de la Ley 27/2007, que reconoce dos lenguas de signos, la española (LSE) y la catalana (LSC), y garantiza una serie de derechos lingüísticos a este colectivo. Desde la perspectiva de la planificación del corpus, las entidades arriba citadas (CNSE, Fundación CNSE, CNLSE y algunas federaciones autonómicas) han promovido la creación de todo tipo de materiales de apoyo para la enseñanza de la lengua de signos (gramáticas, diccionarios, etc.), de una manera similar a como se hace con las lenguas orales minoritarias, si bien con la particularidad de que, al tratarse de lenguas visoespaciales, resultan mucho más adecuados los soportes que permiten usar imágenes de manera flexible y dinámica.

\section{LA PLANIFICACIÓN DE LAS LENGUAS DE SIGNOS EN ESPAÑA: CONSECUENCIAS DE LA TERRITORIALIDAD}

El modelo de las lenguas cooficiales ha dado una pauta para el reconocimiento normativo, en la medida en que puso a disposición de la comunidad sorda española conceptos relacionados con el estatus, con la creación de un estándar y la aspiración a la normalización en el uso. No obstante, también supone un freno, ya que la regulación lingüística establecida en la Constitución Española se fundamenta en un principio de territorialidad (McRae 1975; Ramallo 2013), de tal manera que se establece la oficialidad del castellano en todo el estado y se traslada a las CCAA la iniciativa a reconocer lenguas cooficiales que lo serán únicamente en el territorio de cada una de ellas. López García-Molins (2012) hace una valoración favorable de los logros de la política lingüística española, basada en el reparto constitucional de competencias (sin dejar por ello de proponer líneas de mejora), en tanto que Caamaño (2015) señala la inexistencia de una política lingüística común en España.

Algunas CCAA, en particular Cataluña y Andalucía, han desarrollado legislación específica en relación con la lengua de signos (Ley 17/2010 y Ley 11/2011, respectivamente) ${ }^{7}$. No obstante, los textos legislativos de ambas presentan diferencias dignas de ser comentadas. La ley catalana afirma en su preámbulo que la LSC es la lengua propia de la comunidad catalana signante, es decir, pone la cuestión lingüística en el centro de atención: "La llengua de signes catalana és la Ilengua de les persones sordes i sordcegues signants de Catalunya" (Ley 17/2010, preámbulo; ver Quer 2012). La LSC no es solo el instrumento de comunicación de las personas sordas que residen en Cataluña, es una lengua propia que se usa en el territorio, y por ese motivo forma parte de los intereses y los objetivos de la política lingüística de la comunidad autónoma: "correspon al departament competent en matèria de política lingüística impulsar la regulació de la llengua de signes catalana com a patrimoni lingüístic català" (Ley 17/2010, preámbulo). En el caso catalán,

\footnotetext{
${ }^{7}$ En Cataluña: Ley 17/2010, de 3 de junio, de la lengua de signos catalana. Diari Oficial de la Generalitat de Catalunya, número 5647, Barcelona, de 10 de junio de 2010; en Andalucía: Ley 11/2011, de 5 de diciembre, por la que se regula el uso de la lengua de signos española y los medios de apoyo a la comunicación oral de las personas sordas, con discapacidad auditiva y con sordoceguera en Andalucía. Boletín Oficial de la Junta de Andalucía, número 244, de 15 de diciembre de 2011.
} 
se puede reconocer un precedente para el reconocimiento de la LSC en el caso de la lengua aranesa, una variedad del occitano que la legislación catalana reconoce como propia del territorio del Valle de Arán ${ }^{8}$. La ley andaluza, por su parte, inscribe la cuestión del reconocimiento normativo de la lengua de signos en el marco del derecho a la igualdad y la accesibilidad, es decir, en el entorno de las acciones orientadas a facilitar las condiciones de vida de las personas con discapacidad.

Otras CCAA han dedicado alguna atención a los derechos lingüísticos de las personas sordas de sus territorios en sus respectivos estatutos de autonomía 9 . Aparecen menciones a la protección de las lenguas de signos (española, catalana, o con la calificación de "propia de los sordos") en los textos estatutarios de Aragón, Castilla y León, Cataluña, Comunitat Valenciana, Illes Balears y Extremadura. Salvo en el caso del Estatuto de Autonomía de Cataluña, en que aparece en un artículo sobre la protección del catalán, en los demás casos estas menciones no se hacen en el marco de los derechos lingüísticos de la ciudadanía, sino en el de la defensa de la igualdad de derechos de las personas con discapacidad.

Lo anteriormente expuesto lleva a una interpretación según la cual en el contexto español la legislación sobre las lenguas de signos se inscribe mayoritariamente en la concepción de la sordera como discapacidad, y solo en Cataluña la cuestión alcanza un tratamiento en el ámbito de las lenguas y la política lingüística.

Ocurre, por otra parte, que la política lingüística de las CCAA con lengua cooficial (de nuevo con la excepción de Cataluña) se entiende y se realiza hacia la lengua cooficial, pero no alcanza a planificar ni dinamizar el uso de la lengua de signos en el territorio, seguramente por una mal entendida interpretación del adjetivo española en el nombre de la LSE.

\section{El caso de Galicia: ¿lengua de signos española o lengua de signos GALLEGA? \\ 6.1. La política lingüística en Galicia y la cuestión de la lengua de signos gallega}

Como se ha dicho más arriba (sección 1), los colegios específicos de sordos eran tradicionalmente el espacio de socialización en el que una buena parte de las niñas y niños sordos aprendían la lengua de signos. El modelo educativo ha cambiado en las últimas décadas hacia un tipo de centro que pretende integrar la diversidad funcional (junto a otras diversidades) en las aulas de los colegios (González Abelaira 2016), lo cual no disminuye la influencia del papel de la escuela en el aprendizaje de la lengua de signos por parte de este colectivo, pero introduce elementos nuevos cuya descripción excede los límites de este trabajo. En los colegios específicos de sordos, el aprendizaje se desarrollaba de manera no planificada, fuera de las aulas, ya que el objetivo de la educación era la oralización o desmutización de la persona sorda ${ }^{10}$. El colegio del que procede una persona sorda determina una dimensión de variación lingüística que debe tenerse en cuenta en los estudios que consideren variables sociolingüísticas de las lenguas de signos, pues cada centro tenía un conjunto de formas léxicas específicas (Parkhust / Parkhust 2000). En el caso de Galicia es posible identificar un acervo léxico propio (Caamaño Hermida 2008a,b), que se conformó en torno a los colegios de sordos de Santiago de Compostela (fundado en 1864), Lugo

\footnotetext{
${ }^{8}$ La protección al aranés aparece ya para el territorio del Valle de Arán en la Ley 7/1983, de 18 de abril, de normalización lingüística en Cataluña. Los derechos lingüísticos de los araneses se consolidan en la Ley 16/1990, de 13 de julio, sobre régimen especial del Valle de Arán, donde se declara la oficialidad de la lengua propia en el territorio. Posteriormente, la Ley 35/2010, de 1 de octubre, del occitano, aranés en Arán, regula la enseñanza del aranés en Arán, así como su uso, fomento y difusión. Además, esta ley garantiza la proyección exterior del aranés, con especial mención a otros territorios de lengua occitana, así como su protección en el ámbito catalán. La Ley 1/2015 del Valle de Arán ratifica la oficialidad de la lengua aranesa en toda Cataluña.

${ }^{9}$ La página web de la CNSE (www.cnse.es) cuenta con un apartado de legislación de interés, estatal y autonómica.

${ }^{10} \mathrm{~A}$ pesar de ello, los signos tenían un carácter instrumental, y eran empleados como apoyo para facilitar la comprensión. González Abelaira (2016) hace referencia a los intentos de planificar un sistema de signos común a los diferentes colegios de sordos, lo cual da idea de la importancia que tenían para la comunicación en el colegio.
} 
(1954), O Grove (1952) y A Coruña (1964) (González Abelaira 2016). Es interesante destacar que las dos publicaciones que vieron la luz con este léxico de la variedad gallega (Caamaño Hermida $2008 \mathrm{a}, \mathrm{b})$ no se refieren a ella como lengua de signos gallega; antes bien, utilizan la expresión "lengua de signos en gallego", que parece sugerir una matizada relación entre los signos y el ser gallego, de tal forma que solo sería gallega la equivalencia de las unidades léxicas incorporadas y su definición, que en efecto se presenta en gallego escrito.

La política lingüística en Galicia se sustenta en el artículo 3 de la Constitución española y el Estatuto de Autonomía de Galicia, aprobado en 1981, que define el gallego como la lengua propia de Galicia, dispone la cooficialidad del gallego y el castellano y reconoce el derecho de usar y conocer la lengua gallega en el territorio. La Lei 3/1983, do 15 de xuño, de Normalización Lingüística pone las bases para el desarrollo de una política lingüística que permita incorporar el gallego a la enseñanza, la administración y los medios de comunicación. La publicación en el año 2010 del Decreto 79/2010, do 20 de maio, para o plurilingüismo no ensino non universitario de Galicia introduce cambios en la enseñanza de las lenguas en la enseñanza no universitaria, a partir del argumento de que las sociedades globalizadas exigen a los individuos el conocimiento de lenguas extranjeras:

a realidade social europea en que vivimos, nun contexto de globalización e de mobilidade laboral, sitúanos nun espazo internacional de plurilingüismo. Esta nova realidade esixe un marco educativo que atenda esta necesidade social, posibilitando a capacitación efectiva do alumnado nas dúas linguas oficiais e nunha ou varias linguas estranxeiras (Preámbulo)

En lo que respecta a las lenguas de signos, este marco legislativo podría constituir un elemento que facilitara su inclusión en los niveles educativos a los que se refiere el decreto, pues parte de un modelo de sociedad lingüísticamente diversa. Esta es la premisa en la que se sustenta González Abelaira (2017) para presentar una propuesta de "modelo de educación plurilingüe e inclusivo para Galicia", como parte de su tesis de doctorado. La autora defiende que la idea de plurilingüismo, unida a un compromiso ético hacia la diversidad lingüística, podría conducir a la introducción de la lengua de signos en aquellos centros educativos donde hay alumnado sordo, habida cuenta de que el mencionado decreto deja a los centros educativos un margen de maniobra para adaptarse a su situación contextual. No obstante, el decreto solo reconoce como lenguas maternas del alumnado el español y el gallego (las lenguas cooficiales), de tal manera que se garantiza la competencia oral y escrita en ambas. En la práctica, las acciones por parte de la administración gallega tienden a reforzar un modelo de planificación lingüística sustractivo que impide la incorporación de las lenguas de signos en el ámbito de la educación ${ }^{11}$.

La pesquisa desarrollada por González Abelaira (2016) plantea en un medio académico una pregunta que desde la administración gallega había sido formulada por Modesto Rouco Villar, subdirector general de Promoción de la Autonomía Personal de la Xunta de Galicia (González Abelaira 2016: $97-98)^{12}$ : ¿existe una lengua de signos gallega? La autora presenta un panorama en el que desde Galicia los líderes sordos habrían optado mayoritariamente por un modelo centralista de planificación lingüística de la LSE (González Abelaira 2017: capítulo 6). Su investigación constituye un hito en el medio académico, que en Galicia se venía ocupando desde hace

\footnotetext{
${ }^{11}$ Sirva como ejemplo la retirada del apoyo, en el curso 2017-2018, a la asignatura optativa de lengua de signos española que se venía impartiendo en el CEIP Escultor Acuña de Vigo desde 2012-2013. Esta situación fue objeto de una proposición no de ley, formulada por el Bloque Nacionalista Galego (BNG) ante la Mesa del Parlamento de Galicia, registrada el 28 de diciembre de 2017 (ver Boletín Oficial do Parlamento de Galicia, X lexislatura, n² 236, 10 de xaneiro de 2018, disponible en http:// www.es.parlamentodegalicia.es/sitios/web/BibliotecaBoletinsOficiais/B100236_1.pdf, p. 102, con acceso 10/03/2018). La mayoría parlamentaria del Partido Popular bloqueó la posibilidad de reactivar la asignatura por parte del colegio. Los argumentos de la administración autonómica para no dotar la plaza de docente de LSE se basan en que la plantilla de especialistas en Audición y Lenguaje (AL) del centro es suficiente para atender las necesidades del alumnado, pero la figura de $\mathrm{AL}$ tiene la función de rehabilitar y enseñar la lengua oral, y no se les requiere conocimientos de lengua de signos.

${ }^{12}$ La autora menciona una reunión convocada en 2012 por el subdirector general de Promoción de la Autonomía Personal de la Xunta de Galicia, con el objeto de valorar las posibilidades de desarrollar en Galicia la Ley 27/2007. En ella el convocante preguntó, ante varios colectivos de sordos, padres de sordos, logopedas e intérpretes, si existía una lengua de signos gallega. Solo FeGaXor, una entidad de sordos que no sigue la línea oficial de FAXPG, contestó en sentido afirmativo. En la actualidad, la Asociación de Personas Sordas de Santiago de Compostela (APSSC) apoya a tres de sus socios en la consecución del reconocimiento de la LSG (testimonio de su presidente, José A. Pais Freire).
} 
décadas de la investigación sobre la lengua de signos sin cuestionar las directrices de los líderes del movimiento asociativo sordo en lo que se refiere a la reivindicación del estatus o la elaboración del corpus de la variedad gallega.

La comunidad sorda de Galicia no ha sido del todo ajena a la cuestión de si se debe o no reconocer la lengua de signos gallega (LSG). En el año 2016, según testimonio (a través de un correo electrónico) del presidente de la Asociación de Personas Sordas de Santiago de Compostela (APSSC), José A. Pais Freire, esta entidad local propone a la Federación de Asociacións de Persoas Xordas de Galicia (FAXPG) abrir el debate en torno a la LSG. La junta directiva de FAXPG acuerda que "por el momento, no se va a continuar con el procedimiento para el reconocimiento de la lengua de signos gallega (LSG) mediante trámite parlamentario". Se aduce que existen otros objetivos prioritarios y que faltan estudios de calidad que avalen la LSG. El tema ya se trató con anterioridad sin que se obtuvieran pruebas suficientes para defender la propuesta y faltan medios humanos y económicos.

\subsection{La postura oficial de la FAXPG}

En el contexto de nuestra reflexión sobre las consecuencias del modelo territorial español en la activación de políticas lingüísticas que protejan y promuevan las lenguas de signos, juzgamos necesario conocer de manera directa la postura de la FAXPG, entidad que representa mayoritariamente a las personas sordas de Galicia, que había respondido negativamente a la pregunta sobre la existencia de la lengua de signos gallega ${ }^{13}$. FAXPG es una organización no gubernamental que representa los intereses de las personas sordas. Más concretamente, desarrolla acciones encaminadas a mejorar su acceso a la información. También organiza actividades formativas (en particular para enseñar la LSE) y cuenta con un programa de sensibilización y difusión. Se le puede considerar agente de planificación lingüística por sus acciones hacia las administraciones gallegas ${ }^{14}$.

En la actualidad la labor de reivindicación de los derechos lingüísticos es responsabilidad del CNLSE, órgano de ámbito estatal. La Federación gallega colabora a través de sus representantes, pero no tiene un papel singularizado en la lucha política, si bien se puede afirmar que sí fue más activa en este sentido en los años anteriores a 2007. A través de las asociaciones locales (siete en Galicia) se organizan actos de reivindicación de la lengua de signos en fechas señaladas, como es el Día Nacional de las Lenguas de Signos Españolas, que se celebra el 14 de junio. Las denominaciones que emplea FAXPG (y que se usan mayoritariamente entre las personas sordas gallegas) son lengua de signos española, LSE o (genéricamente) lengua de signos.

Con el objetivo de conocer la postura de FAXPG hacia la lengua de signos gallega solicitamos una reunión con su presidente, Iker Sertucha Lista, que ejerce este cargo desde 2012 y es además presidente del Comité de Entidades Representantes de Personas con Discapacidad (CERMI) en Galicia desde 2016. Es una persona sorda con formación universitaria en Sociología. Como presidente de FAXPG ha colaborado en un volumen sobre educación con un capítulo en el que contextualiza los problemas de la educación de las niñas y niños sordos en Galicia y describe los recursos existentes, incluidos los servicios que ofrece la entidad que preside (Sertucha Lista 2016).

El presidente nos recibió en la sede de la entidad, en A Coruña, el día 6 de junio de 2017. La reunión se había preparado previamente para que tuviera la forma de una entrevista estructurada en torno a un guion que incluía preguntas sobre las siguientes cuestiones:

- Percepción por parte del presidente de la existencia de una variedad gallega

- Nombre de la lengua de signos que hablan las personas sordas de Galicia

- Si es necesario el reconocimiento normativo de una lengua de signos gallega

- Qué lengua(s) de signos han de aprender en el colegio las niñas y niños sordos

\footnotetext{
${ }^{13}$ También desarrollamos gestiones para entrevistar a alguna persona que pudiera dar un punto de vista diferente, pero no logramos ningún resultado, por lo que este estudio se circunscribe a presentar la postura oficial de FAXPG.

${ }^{14}$ Ver memoria anual FAXPG de 2016 en la página web de FAXPG https://drive.google.com/file/d/OB4itoohbkyEJaWZ4OUJ5aUdJdkk/view (con acceso 24/02/2018).
} 
- Qué lengua o sistema es adecuado para la comunicación internacional de las personas sordas

- Qué lengua o sistema de comunicación internacional deben aprender las niñas y niños sordos

- Qué acciones de política lingüística es oportuno emprender para mejorar las condiciones de vida de las personas sordas

El guion se había elaborado de tal manera que las preguntas se pudieran formular en lengua de signos, y las respuestas fueran grabadas para su análisis posterior. Pero este plan hubo de ser adaptado a las condiciones reales de la entrevista, pues no se obtuvo permiso para grabar y se realizó con la mediación de intérpretes. No obstante, se trataron todos los temas que interesaban a los investigadores y, una vez concluida la reunión, se tomó nota de las respuestas que se habían retenido de forma memorística. El hecho de que se haya tenido que optar por esta metodología implica que se haya de renunciar a transcribir fragmentos de la entrevista, pues no hay un registro fiable de la misma que permita desarrollar esta práctica.

El encuentro se realizó en un tono cordial y colaborativo. En el preámbulo se clarificaron los objetivos de la investigación y se precisó que la finalidad era conocer las opiniones del presidente de FAXPG en tanto que líder de la comunidad sorda gallega. El entrevistado respondió a las preguntas desde tal posición y de forma clara. A continuación se resumen las respuestas.

\subsubsection{Sobre la oportunidad de reconocer una lengua de signos gallega}

El presidente manifiesta no tener opiniones en contra de una posible lengua de signos gallega (LSG), y desde la FAXPG la apoyaría en caso de que estudios de investigación documentaran bien su gramática y su léxico, pero no va a apoyar esa tarea. Repite varias veces a lo largo de la reunión que no es una prioridad. Hace unos tres años, una persona sorda ${ }^{15}$ presentó una propuesta a FAXPG sobre la LSG, se le atendió y se le explicó que no es prioritario y que requiere que se documente antes de ser reconocida.

\subsubsection{Sobre las lenguas de signos en el sistema educativo}

Este tema se trata de manera conjunta con el relativo a la comunicación internacional, de tal manera que la pregunta que se le plantea al presidente de FAXPG es qué lenguas (orales y de signos) querría que aprendieran las niñas y niños sordos en una situación ideal en que en Galicia se implantase una política lingüística más favorable a la enseñanza de las lenguas de signos en la escuela. Al enunciar la pregunta mencionamos las lenguas de signos española (LSE), gallega (LSG), norteamericana (ASL) y también el sistema internacional de signos (SIS), un código mixto y fuertemente dependiente del contexto empleado en reuniones y congresos internacionales de personas sordas (Hiddinga / Crasborn 2011).

La postura del entrevistado ante el SIS no es favorable porque es un recurso, no una lengua de signos, y no es adecuado que los niños la aprendan. Este juicio apunta a una cierta falta de confianza en las posibilidades del SIS como medio de comunicación estable, en la medida en que no es una lengua en sentido estricto (Hiddinga / Crasborn 2011: 493). En cuanto al debate LSE-LSG, razona que los intérpretes usan la LSE, y esto podría crear dificultades de comprensión a los niños sordos cuyas familias les hablaran en LSG. Para Iker Sertucha es necesario que las niñas y niños sordos aprendan LSE y que accedan a la lectura. No menciona el aprendizaje de otras lenguas de signos. Del aprendizaje del inglés dice que también es un recurso, lo cual entendemos en el sentido de que valora su carácter instrumental como lengua internacional pero tal vez desconfíe de su utilidad en el contexto de la educación de personas sordas.

A pesar de que se le sugiere que, de manera similar a como ya se hace en un colegio de Vigo (en el momento de la entrevista) en el que los niños y niñas aprenden el inglés y la LSE sin que sea un problema, se podría implementar un aprendizaje de inglés y lengua de signos británica

${ }^{15}$ Imaginamos que se trata de Andrés Caamaño Hermida, pero no cita ningún nombre. 
como lenguas extranjeras para el alumnado sordo, discrepa de este parecer, y se mantiene en la idea de enseñar una única lengua de signos, la LSE.

\subsubsection{Sobre las necesidades de la comunidad sorda gallega en términos de política lingüística}

Las necesidades de la comunidad sorda, en términos de política lingüística, se centran en dos pilares: la interpretación y la figura del experto en lengua de signos, cuya formación aún no está regulada. Es lo que defiende desde su puesto de presidente, adoptando una postura de pragmatismo. No es función de la federación decidir sobre una lengua de signos u otra, sino atender a los problemas del día a día.

Es preciso puntualizar que las prioridades señaladas por lker Sertucha coinciden con dos acciones que se promovieron desde el CNLSE, consistentes en sendos estudios sobre la formación de intérpretes y de especialistas en LSE, en forma de grado universitario, que se materializaron en la publicación de informes (Esteban et al. 2015a y 2015b).

Como el presidente hace referencia a acciones impulsadas por el CNLSE, se le sugiere que en Galicia la lengua de signos es tratada como un asunto de política social y de igualdad, pero no trabajan sobre ella los departamentos que en el gobierno autonómico se ocupan de política lingüística y de educación. Este comentario no le suscita una opinión, por lo que se deduce que el presidente de FAXPG asume que para la LSE la política lingüística se haga de forma centralizada, desde Madrid, renunciando a un tratamiento territorial.

\section{RECAPITULACIÓN Y REFLEXIÓN FINAL}

La revisión presentada en este artículo de la política lingüística que se desarrolla en España en relación con las lenguas de signos se resume en los siguientes términos:

- Cataluña desarrolla una política lingüística que promueve y difunde el catalán hablado y la LSC. Solo en esta comunidad autónoma el reconocimiento de la lengua de signos ha ido parejo a la cuestión de los derechos lingüísticos, en la medida en que la reivindicación de la comunidad sorda ha entroncado con el sentimiento nacional catalán.

- Fuera de Cataluña, el tratamiento de las cuestiones relacionadas con la(s) lengua(s) de signos han tenido un tratamiento más ligado al contexto de la discapacidad y los servicios sociales que al ámbito de las lenguas y la política lingüística.

- El modelo lingüístico reconocido en la Constitución Española y en los estatutos de autonomía se basa en el principio de territorialidad.

- Puesto que solo Cataluña tiene reconocida una lengua de signos propia, la lengua de signos de las personas sordas que residen en otras CCAA es la LSE.

- Centrándonos en el caso gallego, la postura oficial de la entidad que cuenta con más poder de representación de la comunidad sorda gallega manifiesta, a través de su presidente, una actitud poco favorable a intervenir activamente en el reconocimiento de una lengua de signos gallega y a elaborar un corpus de esa lengua. No se encuentra en su discurso ningún indicio de que el elemento gallego pueda funcionar como un rasgo diferenciador de carácter simbólico.

- La defensa del reconocimiento de una lengua de signos gallega es una iniciativa de un sector minoritario de la comunidad sorda de Galicia. Solo en el ámbito de la Asociación de Personas Sordas de Santiago de Compostela hemos localizado alguna información en referencia a esa demanda.

- A pesar de que las competencias en política lingüística están transferidas a la Xunta de Galicia, no se aprecia un interés por reivindicar que las acciones en esta materia se extiendan más allá de la lengua gallega oral, para proteger también a la lengua de signos (gallega o española) usada en el territorio. Esto supone derivar esta responsabilidad únicamente en el CNLSE, organismo de carácter estatal creado al amparo de la Ley 27/2007 con la misión de velar por la LSE. Esto no obsta, por supuesto, para que se demanden servicios al gobierno autonómico. 
La elección en favor de la LSE como lengua de la comunidad sorda gallega puede sustentarse en un argumento de pragmatismo: en ausencia de una base social sólida que reivindique el valor simbólico del elemento gallego propio en la variedad lingüística utilizada por las personas sordas que viven en Galicia, se suman fuerzas en una causa común de carácter estatal, lo cual contribuye a visibilizar la LSE como lengua de referencia de las personas sordas de todos los territorios, excluida Cataluña.

Esta decisión no debería ser impedimento para que la Xunta de Galicia proteja la lengua de signos que utiliza la comunidad sorda gallega, tenga esta o no el distintivo de gallega en su nombre. No debería cejar en el empeño de elaborar un texto normativo que ampare los derechos lingüísticos de las personas sordas en los diferentes ámbitos (educativo, sanitario, medios de comunicación, etc.), de manera similar a como lo han hecho ya Cataluña y Andalucía. Se requiere que las administraciones públicas estén a la altura de las demandas de la comunidad sorda, vengan estas canalizadas a través del CNLSE o bien se trate de necesidades que deban ser atendidas desde los gobiernos autónomos, en el ámbito de sus competencias.

\section{Agradecimientos}

Este artículo se ha realizado al amparo de una investigación financiada por el Ministerio de Economía y Competitividad del gobierno español (FFI2013-41929-P; FFI2016-78194-P) y por la Consellería de Cultura, Educación e Ordenación Universitaria de la Xunta de Galicia (R2014/007; ED341D R2016/011; GPC2014/070).

Agracedemos la colaboración del presidente de FAXPG, Iker Sertucha, y del presidente de la APSSC, José A. Pais.

\section{Referencias Bibliográficas}

Alonso Baixeras, Pilar et al. (2003): Libro Blanco de la Lengua de Signos Española en el Sistema Educativo. S.I.: CNSE. Disponible en línea: http://www. fundacioncnse.org/imagenes/Las\%20portadas/pdf/libro_blanco.pdf [19/07/2017].

Baker-Shenk, Charlotte L. / Dennis Cokely (1980): American Sign Language: A Teacher's Resource Text on Grammar and Culture. Washington, D.C: Gallaudet University Press.

Caamaño, Francisco (2015): "¿Justicia sin lenguas o lenguas sin justicia?", Revista $\mathrm{Ca}$ talana de Dret Públic 50, 42-56. http://doi. org/10.2436/20.8030.01.43.

Caamaño Hermida, Andrés (2008a): As nosas mans: Revista de Lingua de Signos en Galego 1. http:// www.lingua.gal/o-galego/promovelo/equipos-de-dinamizacion-da-lingua-galega/recursos-didacticos? content=/Portal-Lingua/ Contido/recursos/equipos-dinamizacion/contido_0788.html [24/02/2018].

Caamaño Hermida, Andrés (2008b): As nosas mans: Revista de Lingua de Signos en Galego 2. http://www.lingua.gal/o-galego/promovelo/ equipos-de-dinamizacion-da-lingua-galega/ recursos-didacticos?content=/Portal-Lingua/ Contido/recursos/equipos-dinamizacion/contido_0788.html [24/02/2018].

Cabeza Pereiro, María del C. / Fernando Ramallo (2016): "Lenguas de signos y educación en España: Una aproximación desde la comunidad sorda", Language Problems and Language Planning 40 (1), 1-25. http://doi.org/10.1075/ Iplp.40.1.01cab

De Meulder, Maartje (2015): "The Legal Recognition of Sign Languages", Sign Language Studies 15 (4), 498-506. doi: http://doi.org/10.1353/ sls.2015.0018.

De Meulder, Maartje (2017): “Promotion in times of endangerment: the Sign Language Act in Finland", Language Policy 16(2), 189-208. http://doi. org/10.1007/s10993-016-9403-5.

Esteban, María Luz et al. (2015a): Propuesta básica de formación universitaria de intérpretes de lengua de signos española y guías-intérpretes de personas sordociegas. Madrid: CNLSE; Real Patronato sobre Discapacidad. http://www.siis.net/ documentos/documentacion/Propuesta\%20 basica\%20de\%20formacion\%20universitaria. pdf [19/07/2017].

Esteban, María Luz et al. (2015b): Informe sobre el perfil profesional del especialista en lengua de signos española. Madrid: CNLSE; Real Patronato sobre Discapacidad. 
Ferreiro, Emilio / Eva Aroca (2008): "Cambio cultural, normalización lingüística de la lengua de signos española", en Antonio Moreno Sandoval (ed.), El valor de la diversidad (meta)lingüística: Actas Del VIII Congreso de Lingüística General. [S. l.: s.n.], 690-700. http://www.lllf.uam.es/clg8/ [19/07/2017].

González Abelaira, Cristina (2016): “Unha lingua de signos galega?", Estudos de Lingüistica Galega 8, 89-106. doi:10.15304/elg.8.3065. http://www. usc.es/revistas/index.php/elg/article/view/3065 [11/04/2017].

González Abelaira, Cristina (2017): Planificación lingüistica de la lengua de signos en el ámbito educativo de la comunidad autónoma de Galicia: perspectivas histórica y émica. Universidade de Santiago de Compostela. Tesis de doctorado inédita.

Hiddinga, Anja / Onno Crasborn (2011):"Signed languages and globalization", Language in Society 40 (4), 483-505.

Jarque, Maria Josep (2012): "Las lenguas de signos: Su estudio científico y reconocimiento legal", Anuari de Filologia. Estudis de lingüística 2, 33-48.

Liñares Barbeito, Xosé L. (2003): "Apuntes para una sociología de la comunidad sorda", Educación y Biblioteca 138, 50-61.

López García-Molins, Ángel (2012): "La política lingüística del estado", en Henrique Monteagudo (ed.), Linguas, sociedade e política. Un debate multidisciplinar. Santiago de Compostela: Consello da Cultura Galega, 171-196.

Marco, Jaime / Sagrario Matéu (2003): Libro blanco sobre hipoacusia. Detección precoz de la hipoacusia en recién nacidos. Madrid: CODEPEH; Ministerio de Sanidad y Consumo. https://www. msssi.gob.es/profesionales/saludPublica/prevPromocion/maternolnfantil/docs/hipoacusia. pdf [19/07/2017].

Massone, María I. / Alejandro Fojo (2011):“Problemática de la estandarización en las lenguas de señas del Río de la Plata", en Luis E. Behares (comp.), $V$ Encuentro Internacional de Investigadores de Políticas Lingüísticas. Montevideo: Asociación de Universidades Grupo Montevideo, 83-89.
McRae, Kenneth D. (1975): "The Principle of Territoriality and the Principle of Personality in Multilingual States", International Journal of the Sociology of Language 4, 33-54. doi: http://doi.org/ 10.1515/ijsl.1975.4.33.

Mitchell, Ross E. / Michael A. Karchmer (2004): "Chasing the mythical ten percent: Parental hearing status of deaf and hard of hearing students in the United States", Sign Language Studies 4 (2), 138-163.

Morales, Esperanza (2008): "La llengua de signes com a vehicle de comunicació i de capital simbòlic", en Àngels Massip (coord.), Llengua i identitat. Barcelona: Publicacions i Edicions de la Universitat de Barcelona, 29-36.

Parisot, Anne-Marie / Julie Rinfret (2012): "Recognition of Langue des Signes Québécoise in Eastern Canada", Sign Language Studies 12 (4), 583-601. doi: http://doi.org/ 10.1353/sls.2012.0013.

Parkhust, Steve / Diane Parkhust (2000): "La variación en las lenguas de signos: un estudio de causas y una metodología analítica", en Francisco Martínez et al., Apuntes de Lingüística en Lengua de Signos Española, Madrid: CNSE, 221-246.

Quer, Josep (2012): "Legal Pathways to the Recognition of Sign Languages: A Comparison of the Catalan and Spanish Sign Language Acts", Sign Language Studies 12 (4), 565-582. http://doi. org/10.1353/sls.2012.0012.

Ramallo, Fernando (2013): “Lenguas de España: territorios, educación, políticas e ideologías", en Francisco Moreno-Fernández / Fernando Ramallo, Las lenguas de España a debate. Valencia: Uno y cero ediciones, 12-45.

Romaine, Suzanne (2008): "The Bilingual and Multilingual Community", en Tej K. Bhatia / William C. Ritchie (eds.), The Handbook of Bilingualism. Londres: Blackwell, 385-405.

Sertucha Lista, Iker (2016): "La atención a la diversidad: ¿nuestra asignatura pendiente?", en $\mathrm{M}^{\mathrm{a}}$. Dolores Fernández Tilve / Gonzalo Francisco Fernández Suárez (coords.), La escuela de ayer, hoy y mañana. Claves y desafíos. Madrid: Dykinson, 117-126. 
